\title{
Christophe Doche
}

\section{Zhang-Zagier heights of perturbed polynomials}

Journal de Théorie des Nombres de Bordeaux, tome 13, no 1 (2001), p. $103-110$

<http://www.numdam.org/item?id=JTNB_2001_13_1_103_0>

(C) Université Bordeaux 1, 2001, tous droits réservés.

L'accès aux archives de la revue « Journal de Théorie des Nombres de Bordeaux » (http://jtnb.cedram.org/) implique l'accord avec les conditions générales d'utilisation (http://www.numdam.org/conditions). Toute utilisation commerciale ou impression systématique est constitutive d'une infraction pénale. Toute copie ou impression de ce fichier doit contenir la présente mention de copyright.

\section{Numdam}

Article numérisé dans le cadre du programme

Numérisation de documents anciens mathématiques

http://www.numdam.org/ 


\title{
Zhang-Zagier heights of perturbed polynomials
}

\author{
par ChRISTOPHE DOCHE
}

RÉSUMÉ. Dans un précédent article, nous étudions le spectre de la hauteur de Zhang-Zagier [2]. Les progrès accomplis reposaient sur un algorithme qui donnaient des polynômes possédant une petite hauteur. Ici, nous décrivons un nouvel algorithme qui produit des hauteurs encore plus petites. Ceci nous a permis de mettre en évidence un point d'accumulation inférieur à 1,289735. Cette borne est meilleure que la précédente qui était 1,2916674. Après quelques définitions nous détaillons le principe de l'algorithme, les résultats obtenus et la construction explicite qui mène à cette nouvelle borne.

Abstract. In a previous article we studied the spectrum of the Zhang-Zagier height [2]. The progress we made stood on an algorithm that produced polynomials with a small height. In this paper we describe a new algorithm that provides even smaller heights. It allows us to find a limit point less than 1.289735 i.e. better than the previous one, namely 1.2916674 . After some definitions we detail the principle of the algorithm, the results it gives and the construction that leads to this new limit point.

\section{Introduction}

Let $P$ be a polynomial in $\mathbb{Z}\left[z_{1}, \ldots, z_{n}\right]$. The Mahler measure of $P$ is then defined by

$$
M(P)=\exp \left\{\int_{0}^{1} \ldots \int_{0}^{1} \log \left|P\left(e^{2 i \pi t_{1}}, \ldots, e^{2 i \pi t_{n}}\right)\right| d t_{1} \ldots d t_{n}\right\} .
$$

If $P$ is a one variable polynomial, $P(z)=a_{0} \prod_{j=1}^{d}\left(z-\alpha_{j}\right)$, Jensen's formula ensures that

$$
M(P)=\left|a_{0}\right| \prod_{j=1}^{d} \max \left(1,\left|\alpha_{j}\right|\right) .
$$

In this case we denote the absolute Mahler measure of $P$ i.e. $M(P)^{1 / d}$ by $\mathfrak{M}(P)$. If $\alpha \in \overline{\mathbb{Q}}$, we agree that $M(\alpha)$ and $\mathfrak{M}(\alpha)$ represent respectively the Mahler measure and the absolute Mahler measure of the irreducible 
polynomial of $\alpha$ with coefficients in $\mathbb{Z}$. The Zhang-Zagier height or simply the height of $\alpha$, denoted by $\mathfrak{H}(\alpha)$, is then defined as $\mathfrak{H}(\alpha)=\mathfrak{M}(\alpha) \mathfrak{M}(1-\alpha)$. From results of Zhang and of Zagier (cf. [10], [9]), we know that if $\alpha$ is an algebraic number different from the roots of $\left(z^{2}-z\right)\left(z^{2}-z+1\right)$,

$$
\mathfrak{H}(\alpha) \geqslant \sqrt{\frac{1+\sqrt{5}}{2}}=1.2720196 \ldots
$$

In fact Zagier gives also the algebraic numbers which lead to an equality in (3). They are exactly the roots of $\Phi_{10}(z) \Phi_{10}(1-z)$ where $\Phi_{10}(z)$ represents the 10th cyclotomic polynomial.

Our contribution to the study of the Zhang-Zagier height consisted firstly of an improvement of (3), namely

Theorem A. Let $\alpha$ be an algebraic number. We suppose that $\alpha$ is different from the roots of $\left(z^{2}-z\right)\left(z^{2}-z+1\right) \Phi_{10}(z) \Phi_{10}(1-z)$. Then

$$
\mathfrak{H}(\alpha) \geqslant 1.2817770214 .
$$

Secondly, we proved that $\mathcal{V}=\{\mathfrak{H}(\alpha) \mid \alpha \in \overline{\mathbb{Q}}\}$ admitted a limit point less than 1.2916674. Finally, we discovered an algebraic integer whose height, $1.2875274 \ldots$, is less than the smallest previously known i.e. $1.2903349 \ldots$ [4]. All this work is described in [2].

From this, we are able to do a little better with the help of a new algorithm. In fact the main result of this paper is the following.

Theorem. The smallest limit point of $\mathcal{V}=\{\mathfrak{H}(\alpha) \mid \alpha \in \overline{\mathbb{Q}}\}$ is less than 1.289735 .

Before we prove this theorem, we display some strange relations.

Let

$$
\begin{aligned}
A_{1}(z) & =z \\
A_{2}(z) & =z^{2}-z+1 \\
A_{3}(z) & =\Phi_{10}(z)=z^{4}-z^{3}+z^{2}-z+1 \\
A_{4}(z) & =z^{8}-3 z^{7}+4 z^{6}-2 z^{5}+x^{4}-2 z^{3}+3 z^{2}-2 z+1 \\
A_{5}(z) & =z^{16}-7 z^{15}+23 z^{14}-45 z^{13}+57 z^{12}-46 z^{11}+19 z^{10} \\
& +5 z^{9}-9 z^{8}-10 z^{7}+39 z^{6}-56 z^{5}+52 z^{4}-34 z^{3}+16 z^{2}-5 z+1 .
\end{aligned}
$$

These polynomials are remarkable according to their height. Indeed $A_{1}$ and $A_{2}$ have a trivial height, $\mathfrak{H}\left(A_{3}\right)$ is the second point of the spectrum, $A_{4}$ has the smallest height for the degree 8 and $\mathfrak{H}\left(A_{5}\right)=1.2875274 \ldots$ is 
the smallest known height greater than $1.2720196 \ldots$ We verify that

$$
\begin{aligned}
& A_{2}(z)=-A_{1}(z) A_{1}(1-z)+1 \\
& A_{3}(z)=A_{2}^{2}(z)+A_{1}(z) A_{1}^{2}(1-z) \\
& A_{4}(z)=A_{3}(z) A_{3}(1-z)+A_{1}(z) A_{1}^{2}(1-z) A_{2}^{2}(z) \\
& A_{5}(z)=A_{4}(z) A_{4}(1-z)+A_{1}(z) A_{1}^{2}(1-z) A_{2}^{2}(z) A_{3}(z) A_{3}(1-z) .
\end{aligned}
$$

So we make the hypothesis that small heights come from "perturbed" polynomials of small height.

The phenomenon seems quite general since it occurs also for the Mahler measure [6] and for the spectrum of $\mathfrak{M}(\alpha) \mathfrak{M}(1 /(1-\alpha)) \mathfrak{M}(1-1 / \alpha)$ [3]. Therefore it is quite reasonable to test polynomials $A_{6}(z)$ such that

$$
\begin{aligned}
A_{6}(z)= & A_{5}(z) A_{5}(1-z) \\
& \pm A_{1}^{a_{1}}(z) A_{1}^{a_{2}}(1-z) A_{2}^{a_{3}}(z) A_{3}^{a_{4}}(z) A_{3}^{a_{5}}(1-z) A_{4}(z)^{a_{6}} A_{4}^{a_{7}}(1-z)
\end{aligned}
$$

with the $a_{i}$ 's in $\mathbb{N}$ verifying $a_{1}+a_{2}+2 a_{3}+4\left(a_{4}+a_{5}\right)+8\left(a_{6}+a_{7}\right) \leqslant 31$. The results are quite disappointing since we get only a handful of good polynomials; the best corresponding to the choice

$$
A_{6}(z)=A_{5}(z) A_{5}(1-z)-A_{1}^{5}(z) A_{1}^{5}(1-z) A_{2}^{5}(z) A_{3}(z) A_{3}(1-z)
$$

having $\mathfrak{H}\left(A_{6}\right)=1.2906235 \ldots$ We also notice that $A_{6}(z)$ is invariant under the map $z \mapsto 1-z$. Now, every polynomial of even degree symmetric under $z \mapsto 1-z$ can be expressed in terms of

$$
X=z(1-z)
$$

For instance $\Phi_{10}(z) \Phi_{10}(1-z)=X^{4}-2 X^{3}+4 X^{2}-3 X+1$.

After many unfruitful computations we decided to consider only polynomials in the new variable $X$ and no longer in $z$. As a consequence we can increase the number of perturbing factors. So we put

$$
\begin{aligned}
& P_{1}(X)=X \\
& P_{2}(X)=1-X \\
& P_{3}(X)=X^{3}+X^{2}-2 X+1 \\
& P_{4}(X)=X^{4}-2 X^{3}+4 X^{2}-3 X+1
\end{aligned}
$$




$$
\begin{aligned}
& P_{5}(X)= X^{8}-2 X^{7}+4 X^{6}-7 X^{5}+13 X^{4}-16 X^{3}+12 X^{2}-5 X+1, \\
& P_{6}(X)= X^{8}-3 X^{7}+8 X^{6}-16 X^{5}+26 X^{4}-27 X^{3}+17 X^{2}-6 X+1, \\
& P_{7}(X)= X^{12}-3 X^{11}+8 X^{10}-18 X^{9}+36 X^{8}-62 X^{7}+97 X^{6} \\
&-123 X^{5}+114 X^{4}-73 X^{3}+31 X^{2}-8 X+1 \\
& P_{8}(X)= X^{12}-3 X^{11}+7 X^{10}-14 X^{9}+30 X^{8}-58 X^{7}+96 X^{6} \\
&-123 X^{5}+114 X^{4}-73 X^{3}+31 X^{2}-8 X+1 \\
& P_{9}(X)= X^{16}-4 X^{15}+10 X^{14}-17 X^{13}+26 X^{12}-47 X^{11}+119 X^{10} \\
&-298 X^{9}+592 X^{8}-878 X^{7}+963 X^{6}-780 X^{5}+464 X^{4} \\
&-199 X^{3}+59 X^{2}-11 X+1,
\end{aligned}
$$

and $d_{i}=\operatorname{deg} P_{i}$. Note that $P_{1}(X)=A_{1}(z) A_{1}(1-z), P_{2}(X)=A_{2}(z)$, $P_{4}(X)=A_{3}(z) A_{3}(1-z), P_{5}(X)=A_{4}(z) A_{4}(1-z), P_{9}(X)=A_{5}(z) A_{5}(1-z)$.

Then we choose a starting polynomial $P(X)$ of degree $d$, we take integers $\left(a_{i}\right)_{1 \leqslant i \leqslant 9}$ and ask simply that

$$
\sum_{i=1}^{9} a_{i} d_{i} \leqslant d-1 .
$$

At this point, for each combination of $\left(a_{i}\right)_{1 \leqslant i \leqslant 9}$ verifying (4) we estimate

$$
\mathfrak{H}\left(P(z(1-z)) \pm P_{1}^{a_{1}} P_{2}^{a_{2}} P_{3}^{a_{3}} P_{4}^{a_{4}} P_{5}^{a_{5}} P_{6}^{a_{6}} P_{7}^{a_{7}} P_{8}^{a_{8}} P_{9}^{a_{9}}(z(1-z))\right)
$$

by the method of Graeffe [1]. If this evaluation is rather small we compute precisely its Zhang-Zagier height. Before we did this search, we knew only 9 polynomials whose height is less than 1.29. Now, just for the degree 28 we have more than 120 polynomials with a height less than 1.29. Figures 1 and 2 help us to see the gap we filled.

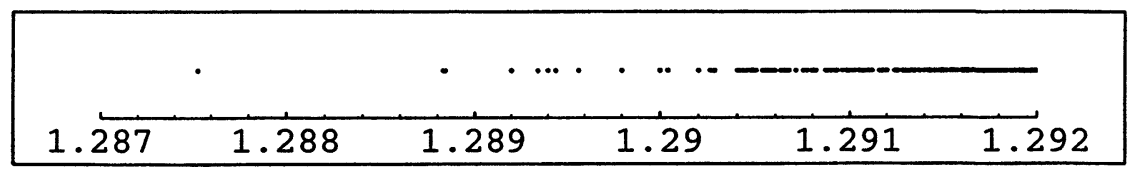

FigURE 1. Known heights previously.

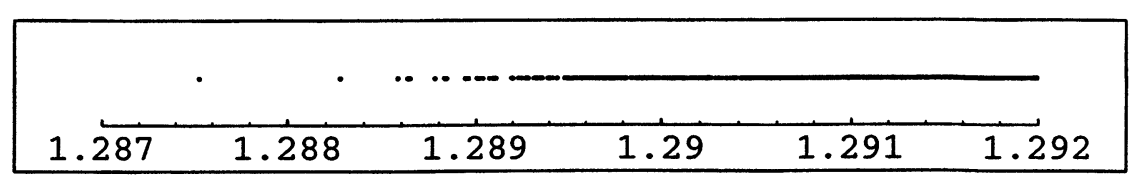

FIGURE 2. Known heights at present. 
Table 1 shows the improvements made, if any, for each degree.

\begin{tabular}{ccc}
\hline \hline Degree & Previous record & New record \\
\hline 10 & $1.2945155 \ldots$ & \\
11 & $1.2939545 \ldots$ & $1.2916397 \ldots$ \\
12 & $1.2888421 \ldots$ & - \\
13 & $1.2926938 \ldots$ & - \\
14 & $1.2917134 \ldots$ & $1.2911770 \ldots$ \\
15 & $1.2914361 \ldots$ & - \\
16 & $1.2875274 \ldots$ & - \\
17 & $1.2907680 \ldots$ & $1.2905866 \ldots$ \\
18 & $1.2913799 \ldots$ & $1.2903115 \ldots$ \\
19 & $1.2926006 \ldots$ & - \\
20 & $1.2893428 \ldots$ & $1.2885499 \ldots$ \\
21 & $1.2904063 \ldots$ & $1.2898748 \ldots$ \\
22 & $1.2913747 \ldots$ & $1.2893658 \ldots$ \\
23 & $1.2917477 \ldots$ & - \\
24 & $1.2888365 \ldots$ & - \\
25 & $1.2893561 \ldots$ & $1.2890842 \ldots$ \\
26 & $1.2909655 \ldots$ & $1.2899285 \ldots$ \\
27 & $1.2901873 \ldots$ & - \\
28 & $1.2895016 \ldots$ & $1.2882759 \ldots$ \\
32 & $1.2907082 \ldots$ & $1.2893407 \ldots$ \\
& & \\
\hline Total & $1.2875274 \ldots$ & - \\
\hline \hline
\end{tabular}

TABLE 1.

It is hard to explain precisely why this algorithm gives good results. Nevertheless here is a kind of heuristic argument. In [2], we observed the importance of the resultant for the Zhang-Zagier height. Namely, a polynomial $P$ with $\mathfrak{H}(P)$ small usually has a small resultant with each of the $P_{i}$. Now it is obvious that

$$
\operatorname{Res}\left(P \pm P_{1}^{a_{1}} P_{2}^{a_{2}} P_{3}^{a_{3}} P_{4}^{a_{4}} P_{5}^{a_{5}} P_{6}^{a_{6}} P_{7}^{a_{7}} P_{8}^{a_{8}} P_{9}^{a_{9}}, P_{i}\right)=\operatorname{Res}\left(P, P_{i}\right)
$$

for any $P_{i}$. So $P \pm P_{1}^{a_{1}} P_{2}^{a_{2}} P_{3}^{a_{3}} P_{4}^{a_{4}} P_{5}^{a_{5}} P_{6}^{a_{6}} P_{7}^{a_{7}} P_{8}^{a_{8}} P_{9}^{a_{9}}$ are good candidates.

These results convince us of the existence of a limit point of $\mathcal{V}$ less than 1.29 which leads us to the Theorem. To prove it, we use the same techniques as in [2]. 


\section{Proof of the Theorem}

With the above notations, we shall use $P_{1}(X), P_{2}(X), P_{4}(X), P_{6}(X)$ and $P_{8}(X)$. We introduce also $Q(X)=Q_{1}(X) Q_{2}(X)$ with

$$
\begin{gathered}
Q_{1}(X)=X^{28}-7 X^{27}+30 X^{26}-97 X^{25}+269 X^{24}-679 X^{23}+1612 X^{22} \\
-3618 X^{21}+7646 X^{20}-15180 X^{19}+28457 X^{18}-50741 X^{17}+86189 X^{16} \\
-138288 X^{15}+206152 X^{14}-279897 X^{13}+339335 X^{12}-360911 X^{11} \\
+331775 X^{10}-260367 X^{9}+172556 X^{8}-95554 X^{7}+43677 X^{6} \\
-16221 X^{5}+4786 X^{4}-1084 X^{3}+178 X^{2}-19 X+1,
\end{gathered}
$$

and

$$
\begin{gathered}
Q_{2}(X)=X^{28}-7 X^{27}+30 X^{26}-96 X^{25}+255 X^{24}-586 X^{23}+1212 X^{22} \\
-2360 X^{21}+4573 X^{20}-9148 X^{19}+18749 X^{18}-37783 X^{17} \\
+71770 X^{16}-124910 X^{15}+195848 X^{14}-273368 X^{13}+335981 X^{12} \\
-359545 X^{11}+331349 X^{10}-260271 X^{9}+172542 X^{8}-95553 X^{7} \\
+43677 X^{6}-16221 X^{5}+4786 X^{4}-1084 X^{3}+178 X^{2}-19 X+1
\end{gathered}
$$

We verify that

$$
\begin{aligned}
\mathfrak{H}\left(P_{1}\right) & =\mathfrak{H}\left(P_{2}\right)=1, & \mathfrak{H}\left(P_{4}\right) & =1.272019650 \ldots, \\
\mathfrak{H}\left(P_{6}\right) & =1.297431163 \ldots, & & \mathfrak{H}\left(P_{8}\right)=1.289442541 \ldots, \\
\mathfrak{H}\left(Q_{1}\right) & =1.288275954 \ldots, & & \mathfrak{H}\left(Q_{2}\right)=1.288646007 \ldots
\end{aligned}
$$

Finally, we need the following lemma proved in [2].

Lemma. Let $P$ be a polynomial in two variables $y$ and $z$, such that $\operatorname{deg}_{z} P>0$. Let $\zeta_{n}$ be $e^{\frac{2 i \pi}{n}}$ and assume that for all $n$ and all $k, P\left(\zeta_{n}^{k}, z\right)$ is not identically zero. We then have

$$
M(P(y, z))^{\left(1 / \operatorname{deg}_{z} P\right)}=\lim _{n \rightarrow \infty} \mathfrak{M}\left(\prod_{k=1}^{n} P\left(\zeta_{n}^{k}, z\right)\right) .
$$

At present let $\left(q_{1}, q_{2}, \ldots, q_{5}\right) \in \mathbb{Q}_{+}^{5}$ and $b$ a denominator of the $q_{i}$ 's. Then it is clear that

$$
\mathfrak{H}\left(\left(P_{1}^{b q_{1}} P_{2}^{b q_{2}} P_{4}^{b q_{3}} P_{6}^{b q_{4}} P_{8}^{b q_{5}}(z(1-z))\right)^{n}-\left(Q^{b}(z(1-z))\right)^{n}\right)
$$

gives rise to a limit point of $\{\mathfrak{H}(P) \mid P \in \mathbb{Z}[z]\}$ when $n$ tends to infinity. Factorizing the polynomial over $\mathbb{C}[z]$, we see that the limit of (5) when $n$ 
tends to infinity is equal to

(6)

$$
\lim _{n \rightarrow \infty} \mathfrak{M}\left(\prod_{k=1}^{n}\left(P_{1}^{b q_{1}} P_{2}^{b q_{2}} P_{4}^{b q_{3}} P_{6}^{b q_{4}} P_{8}^{b q_{5}}(z(1-z))-\zeta_{n}^{k} Q^{b}(z(1-z))\right)\right)^{2}
$$

As the degree in $z$ of the polynomial in the product of (6) is

$$
D(b)=2 b \max \left(56, q_{1}+q_{2}+4 q_{3}+8 q_{4}+8 q_{5}\right),
$$

the Lemma asserts that (6) equals

$$
M\left(P_{1}^{b q_{1}} P_{2}^{b q_{2}} P_{4}^{b q_{3}} P_{6}^{b q_{4}} P_{8}^{b q_{5}}(z(1-z))-y Q^{b}(z(1-z))\right)^{2 / D(b)}
$$

Now recall $(1)$ and put $\chi(s)=e^{2 i \pi s}\left(1-e^{2 i \pi s}\right)$ so that $(7)$ is

$$
\begin{aligned}
{\left[\operatorname { e x p } \left\{\int_{0}^{1} \int_{0}^{1} \log \mid\left(P_{1}^{b q_{1}} P_{2}^{b q_{2}} P_{4}^{b q_{3}} P_{6}^{b q_{4}} P_{8}^{b q_{5}}(\chi(s))\right.\right.\right.} \\
\\
\left.\left.\left.-e^{2 i \pi t} Q^{b}(\chi(s))\right) \mid d s d t\right\}\right]^{2 / D(b)}
\end{aligned}
$$

and finally by $b$ changes of variable $t \mapsto t+j / b$, for $j \in \llbracket 1, b \rrbracket$, we get

$$
\begin{aligned}
{\left[\operatorname { e x p } \left\{\int_{0}^{1} \int_{0}^{1} \log \mid\left(P_{1}^{q_{1}} P_{2}^{q_{2}} P_{4}^{q_{3}} P_{6}^{q_{4}} P_{8}^{q_{5}}(\right.\right.\right.} & (x(s)) \\
& \left.\left.\left.-e^{2 i \pi t} Q(\chi(s))\right) \mid d s d t\right\}\right]^{2 b / D(b)}
\end{aligned}
$$

The trick of considering $z$ as a simple parameter together with formula (2) imply that (8) is nothing but

(9) $[M(Q(z(1-z)))$

$$
\left.\times \exp \left\{\int_{0}^{1} \log ^{+}\left|\frac{P_{1}^{q_{1}} P_{2}^{q_{2}} P_{4}^{q_{3}} P_{6}^{q_{4}} P_{8}^{q_{5}}(\chi(s))}{Q(\chi(s))}\right| d s\right\}\right]^{2 b / D(b)},
$$

where $\log ^{+}(w)$ is $\max (0, \log |w|)$ as usual. Then we search the best $q_{i}$ 's in order to have the smallest limit point possible. As we can easily compute the simple integral of (9) by a Riemann sum, we test several choices of $q_{i}^{\prime} s$. Thanks to these computations, we produce a limit point less than 1.29 thus better than the previously known i.e. 1.2916674. The best combination found is

$$
q_{1}=13.1, q_{2}=10.6, q_{3}=3.2, q_{4}=1.15, q_{5}=0.24
$$

which yields a limit point less than $\ell=1.289735$. Of course this limit point $\ell$ concerns only polynomials but fortunately we can deduce from them a subsequence of irreducible polynomials with a height less than $\ell$ (see $[2$, Lemmas $3,4,5])$. 
Let us say a few words about the polynomials involved in (9). While the selection of $P_{1}, P_{2}, P_{4}, P_{6}, P_{8}$ among $P_{1}, \ldots, P_{9}$ is forced by the search of the minimum of (9), the choice of the polynomials to be perturbed is more arbitrary. In fact, we take $Q_{1}(X)$ and $Q_{2}(X)$ according to their very small height and also because we remarked that a product of $P_{i}$ 's, namely $P_{1}^{7}(X) P_{2}^{5}(X) P_{4}(X)$, divides $Q_{1}(X)-Q_{2}(X)$. This point seems determinant, however we do not understand why it is so important.

The results confirm that our previous algorithm was far from being exhaustive since a great number of heights were missed. Nevertheless, no new height less than 1.2875274 appears, so that we still do not know the second non-trivial point, if any, of $\mathcal{V}$. It would be desirable to improve our knowledge of the set of $\mathfrak{H}(\alpha)$ as it was done for the Mahler measure of non-reciprocal polynomials [7] and for the height of totally real algebraic integers [8] and [5], but we have the feeling that this will be difficult without new ideas.

\section{References}

[1] J. DÉgot, J.-C. HoHL, O. JENVRIN, Calcul numérique de la mesure de Mahler d'un polynome par itérations de Graeffe. C.R. Acad. Sci. Paris 320 (1995), 269-272.

[2] C. Doche, On the spectrum of the Zhang-Zagier height. Math. Comp. 70 (2001), no. 233, 419-430.

[3] G.P. DRESDEN, Orbits of algebraic numbers with low heights. Math. Comp. 67 (1998), 815820.

[4] V. Flammang, Mesures de polynômes. Applications au diamètre transfini entier. Thèse de l'Université de Metz, 1994.

[5] V. Flammang, Two new points in the spectrum of the absolute Mahler measure of totally positive algebraic integers. Math. Comp. 65 (1996), 307-311.

[6] M.J. Mossinghoff, C.G. Pinner, J.D. VAALer, Perturbing polynomials with all their roots on the unit circle. Math. Comp. 67 (1998), 1707-1726.

[7] C.J. SMYTH, On the product of the conjugates outside the unit circle of an algebraic integer. Bull. London Math. Soc. 3 (1971), 169-175.

[8] C.J. SMYTH, On the measure of totally real algebraic integers II. Math. Comp. 37 (1981), 205-208.

[9] D. ZAGIER, Algebraic numbers close both to 0 and 1. Math. Comp. 61 (1993), 485-491.

[10] S. Zhang, Positive line bundles on arithmetic surfaces. Ann. of Math. 136 (1992), 569-587.

Christophe Doche

A2X

Université Bordeaux 1

351, cours de la Libération

33405 Talence Cedex

France

E-mail : cdocheomath.u-bordeaux.fr 